\title{
Benveniste on the Benveniste affair
}

\author{
Dr Jacques Benveniste replies to some of the points raised in recent correspondence in a text which is \\ printed (as on a previous occasion) unchanged except for grammatical reasons.
}

\section{Paris}

WHAT a deluge! Certainly appropriate about a paper on water but a lot of it could have been deleted using Nature's criteria for publication. Our publication of our paper was a cry for help to explain these puzzling results. Instead we got a fraud squad, an unsound (a fraud implicating five laboratories?) insult to respected scientists, who from the start and thereafter were treated as criminals. All scientists on Earth should resent the insult or at least the threat.

The style of the report, some of the expressions used, the sensational titles indeed, it's a shame. A well-done job, admittedly, that condemns us first, then covers pages that discouraged many readers. And, shamelessly, a critical sentence indicating that many (?) of our results are statistically correct was removed at the last minute, after receiving my answer (Nature 334, 291, column 3, paragraph 2).

A section called "Collaboration" was also added at the last minute which is filled with "mistruths": data from Israel, twice described as not available, can be found

in our Nature paper (Table 2), and the corresponding raw data were given to Nature editors in March 1987. The report by Maitre Simart, the bailiff, describing the coding and decoding in April, May and June 1987, in the presence of the dean of our faculty, is available to anyone.

Since, to our surprise, the unbelievable Nature report was taken by some as evidence negating our data, we have to repeat once more the litany of the amateurish mistakes of the "experts" that "do not match their extraordinary claim" of being the world scientific conscience and judge: confusion of single-code done twice with double-blind, with circus-like - and fraud-seeking - pantomime of sticking the tape-armoured code to the ceiling (why not in their pocket since they knew the code? Because they wanted to catch the villains tampering with the sacred paper, hence the "expert at opening envelopes"), participation of the referee in the ball-game (that he had never played before), misquotation in the report of their own figures, erroneous Fig. 6 captions and the immortal "we have a record of the proceedings on an unbroken reel of tape".

Yet the most salient feature of this report is confirmation of our data by two positive experiments (Fig. 2 and Fig. 6) that remain unchallenged. I am supposed not to have ever seen anything such as Fig. 2 before, when the same is in Table 1 and Fig. $1 b$ of our paper. The two plots in Fig. 6 are declared discordant, exactly what is printed in our article.

The following correspondence for weeks occupied precious Nature space to show both that these data do not exist and how to explain them. I am more concerned at Gaylarde's claim that they are synthetic $(334,375)$, which is entirely unsubstantiated as well as being a blow to academic courtesy.

It is clear from the Table 1 legend of our paper that dilutions were double-coded but not the reading. This could explain an unconscious gap-closing between triplicates but not the differences between coded dilutions and controls. The fourth experiment (Fig. 2 of the report), read blind, yielded "unbelievable", "incredible" replicates that cannot be found in the report. Gaylarde's next argument is indeed damning, but to whom? Counts higher than controls were not ignored but, as usual when using percentages, were equated to 100 since it does not make sense to create basophils. We made clear in our paper that statistics were done on actual numbers and not on percentages.

Metzger (ibid.) and Seagrave $(\mathbf{3 3 4}, 559)$ show no numbers over 100 , but are immune from criticism. In the latter letters, and that of Bonini (334, 559), small numbers of experiments are enough to show we are wrong. But our exact experimental design was not reproduced. Cell biologists know that release of mediator is a late event preceded by myriads of intracellular signals. Our test monitors at the granule matrix level cation competition with a positively charged dye. In fact, we have occasionally also seen histamine release but this is hardly reproducible. Using mediator release to confirm our experiment is as sound as reproducing data on muscle ATP by studying contraction.

Several letters propose hypotheses wilder than our data: the antibodymimicking heparin, a mysterious degranulating molecule that yields a rhythmic fluctuation that is not so rhythmic, cavitation, free radicals. . . . Answer to these entertaining fantasies is in Fig. $1 b$ of our paper.

To try to raise the tone of the debate, I will take the goat/unicorn story from Randi and the request of "different edito- rial standards" for extraordinary data from H. Metzger. J. Randi believes that it takes special data to tell a unicorn from a goat. In the experimental process, data must be collected and interpreted independently of the weight and implication of the hypothesis. A goat is a unicorn when a statistically significant number of experiments have shown a unique horn, obviously after Randi has checked that it was not glued with Araldite. If there are two horns, it's a goat, regardless of electron microscopy, NMR imaging or gene splicing.

This is serious matter. Changing the rules of experimental science will first kill fragile data critical to fringe advances and then science as a whole. Let us not depart from this "fundamental principle of scientific objectivity". Finally, some good news. The riddle is solved: somebody came in our backyard and shot the unicorn.

Indeed, only the smile is left ... with a question: why? It seems illogical that having scrutinized the paper for two years, having urged confirmation of our initial work in independent laboratories, which was done in Canada, Israel and Italy. Nature, short of any valid objection, published it hastily, to then go these extreme lengths to ... deNature it. The answer is to be found both in Nature 333, 787, para. 4 , and in the report $(334,287$, para. 5), both of which emphasize warnings against homoeopathy.

Fact twisting, errors, omissions, misquotations and mistruths are symptoms of a crusade. Also revealing is that at the question "why publish before the inquiry", the Nature staff tells interviewers: "Benveniste would have withdrawn the paper". Clearly the plot was to scare the bird out of the bush to shoot at ease. Their indiscriminate fire is typical of their disarray in front of these obviously positive results.

John Maddox (New York Times 28 September) still declares them non-existent, whereas J. Randi now "admits" (Espressor, Lisbon, 1 October) that it is in fact a fraud. A fraud with five laboratories and no results! What on Earth is this mess? Unfortunately, facts are stubborn and so are we. The numerous truth-seeking scientists all over the world, some of them prompted by our paper and the obviously biased inquiry, have intellectual and technical means either to understand the error or to establish this new field. There is more to come.

Jacques Benveniste 